\title{
Thermal Properties of Side Chain Type Liquid Crystalline Copolymer Composed of a Mesogenic Monomer and Non-Mesogenic Chiral Monomer
}

\author{
Takashi Minara, Kazuhiro Nomura, Keisuke Funaki, \\ and Naoyuki KoIDE \\ Department of Chemistry, Faculty of Science, Science University of Tokyo, \\ 1-3 Kagurazaka, Shinjuku-ku, Tokyo 162, Japan
}

(Received July 24, 1996)

\begin{abstract}
Copolymers were synthesized by the copolymerization of a mesogenic monomer containing a flexible spacer with a chiral monomer. Menthol and 2-methyl-1-butanol were used as chiral moieties. Smectic or nematic phases were exhibited in the copolymers containing a mesogenic group fraction above $40-50 \mathrm{~mol} \%$. The mesogenic group fraction in the copolymers exhibiting a smectic phase was dependent on the chemical structure of the chiral moiety. The induced cholesteric phase was not observed for the copolymers. The mesomorphic temperature range of the copolymers was affected by the chemical structure of the chiral moiety. The mesomorphic temperature range for the copolymers with the 2-methyl butyl group was wider than that of the copolymer containing the menthol derivative.

KEY WORDS Liquid Crystalline Polymer / Menthol / 2-Methyl-1-butanol / Smectic Phase / Nematic Phase /
\end{abstract}

Cholesteric liquid crystalline polymers can be obtained by combination of a chiral component and a mesogenic unit in the main chain, like a low molecular cholesteric liquid crystalline compound. Many researchers have reported induced cholesteric phases prepared by the introduction of a chiral component into the nematogenic polymer. ${ }^{1-3}$ In the case of the side chain type liquid crystalline polymers without a spacer between the polymer backbone and mesogenic pendant, the homopolymer with a cholesteryl moiety in the side chain did not always exhibit a cholesteric phase. However, cholesteric polymers were prepared by the copolymerization of a nematogenic monomer and a chiral monomer containing a rigid core. ${ }^{4}$ Thus to find new methods for the cholesteric phase in the side chain type polymers, we investigated the thermal properties of side chain type liquid crystalline copolymers composed of a mesogenic monomer and a chiral non-mesogenic monomer. ${ }^{5}$ We used $d$-, $l$-menthyl, and 2-methyl butyl groups as the chiral moiety, and a benzoate type mesogenic group composed of three phenylene rings in the side chain. The mesogenic group was directly attached to the polymer backbone with no flexible spacer introduced between the mesogenic group and polymer backbone. The induced cholesteric phase was observed for copolymers containing above 40$60 \mathrm{~mol} \%$ of the mesogenic group. The presence of the induced cholesteric phase was not dependent on the chemical structures of the chiral moiety. However, the selective reflection of visible light was observed only for copolymers with the menthyl group. In the case of the copolymers with the 2-methyl butyl group, the selective reflection of visible light was not observed. With regard to the optical texture for the copolymers, a typical fingerprint texture was observed for the methacrylate copolymers with a 2-methyl butyl group, while the methacrylate copolymers with a menthyl group showed no typical optical texture for the cholesteric phase.

In this report, to examine the influence of the flexible spacer introduced between the mesogenic group and polymer backbone in the cholesteric copolymers on the mesomorphic properties and, especially, the exhibition of the cholesteric phase, copolymers were prepared by copolymerization of a chiral monomer and a mesogenic monomer containing six methylene units as the flexible spacer. The thermal properties of copolymers were investigated using polarizing optical microscopy, differential scanning calorimetry (DSC) and X-ray measurements.

\section{RESULTS AND DISCUSSION}

The synthetic route of a mesogenic monomer is shown in Scheme 1. An enantiotropic mesophase was exhibited for the mesogenic monomer with a flexible spacer. The copolymers, as shown in Scheme 2, were synthesized by copolymerization of the mesogenic monomer and chiral monomer. The thermal properties of the copolymers are summarized in Tables I, II, and III. The phase transition temperatures were plotted as a function of the mesogenic monomer fraction in Figures 1, 2, and 3, respectively. The mesogenic monomer fraction of the copolymers was determined by NMR measurements. The feed fraction

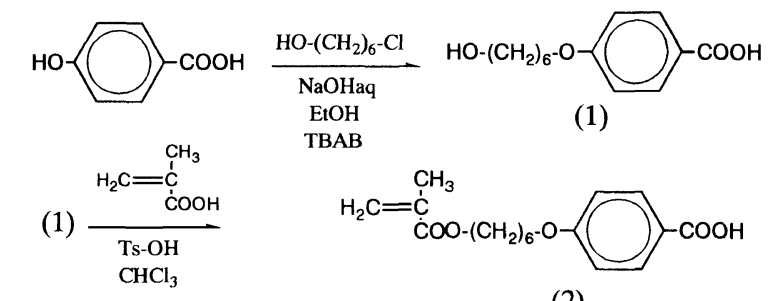

(2)
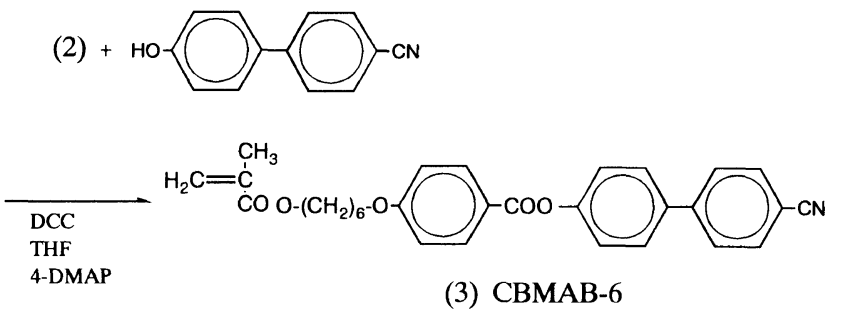

Scheme 1. Synthesis of mesogenic monomer. 
of the mesogenic monomer for the synthesis of the copolymers agreed with the calculated fraction of mesogenic units in the copolymers. The specific rotation for all copolymers increased with increasing the chiral moiety fraction. The values of specific rotations for the chiral homopolymer containing the menthyl groups were larger than that of the chiral monomers, while the specific rotation of the chiral homopolymer with 2-methyl butyl groups was smaller than that of the chiral monomer. The sign of the specific rotation for the copolymer containing

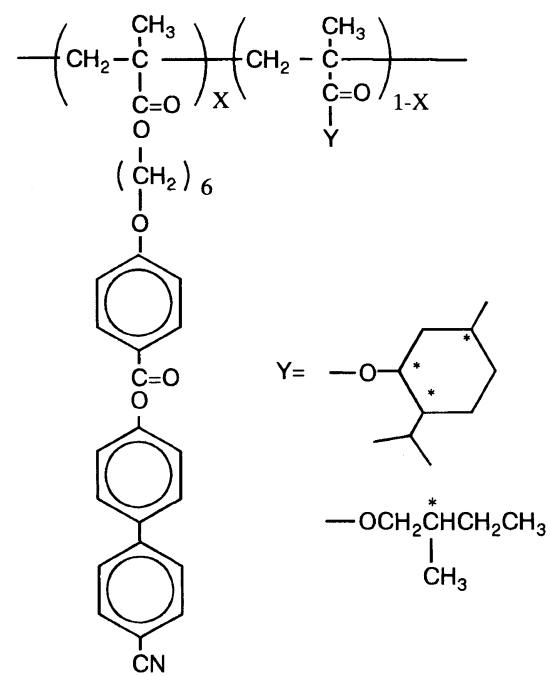

CBMAB

$\begin{array}{ll}\mathrm{Y}=(\mathrm{a})-d & \text { poly(CBMAB-6-co- } d-\mathrm{MtMA})^{*}-\mathrm{x} \\ \mathrm{Y}=\text { (a)- } l & \text { poly(CBMAB-6-co- } l \text {-MtMA) }{ }^{*}-\mathrm{x} \\ \mathrm{Y}=\text { (b) } & \text { poly(CBMAB-6-co-2-MBMA)*-x }\end{array}$

Scheme 2. Structure of side chain type liquid crystalline copolymer. 2-methyl butyl groups depended on the chiral moiety fraction. Mesomorphic properties were observed for the copolymers containing the $d$ - and $l$-menthyl groups as a chiral moiety and above $50 \mathrm{~mol} \%$ of the mesogenic group in the side chain, while the copolymer with the 2-methyl butyl group and above $40 \mathrm{~mol} \%$ of the mesogenic group in the side chain showed mesomorphic properties. No isotropic phase for copolymers with no flexible spacer was observed, clearing temperatures for all copolymers with a flexible spacer were observed. The clearing temperatures for all copolymers with a flexible spacer decreased with decreasing fraction of the mesogenic group in the copolymers. The glass transition temperature did not depend on the fraction of the mesogenic group. Therefore, the mesomorphic temperature range for all copolymers with a flexible spacer decreased with decreasing fraction of the mesogenic group.

In the X-ray diffraction pattern of the poly(4-cyanobiphenyl-4'-[4-(6-methacryloylhexyloxy)benzoate]-co-2(2-methyl-1-butyl methacrylate) ${ }^{*}-69.6$ (poly(CBMAB6-co-2-MBMA)* ${ }^{*} 69.6$ ) as shown in Figure 4 , a sharp peak in the small angle and a broad peak in the wide angle were observed. This indicates that the mesophase structure of the copolymer is a smectic phase. The same X-ray diffraction pattern was observed for the copolymers with a high mesogenic monomer fraction regardless of the chiral moiety. A d-spacing for these copolymers showed a smectic phase with a bilayer structure. The d-spacing for the copolymers decreased with increasing mesogenic monomer fraction regardless of the structure of the chiral moieties. In the case of poly(CBMAB6-co-2-MBMA)* $-x$, a smectic phase was exhibited over the wide range of the mesogenic monomer fraction $(50$ $90 \mathrm{~mol} \%$ ) compared with that for the copolymers with

Table I. Phase transition temperatures, molecular weight, and specific rotation of poly(CBMAB-6-co- $d$-MtMA)*-x

\begin{tabular}{|c|c|c|c|c|c|c|c|c|}
\hline \multicolumn{2}{|c|}{ Mesogenic monomer fraction $/ \mathrm{mol} \%$} & \multirow{2}{*}{\multicolumn{4}{|c|}{ Phase transition temp $/{ }^{\circ} \mathrm{C}$}} & \multirow{2}{*}{$\bar{M}_{n} / 10^{4}$} & \multirow{2}{*}{$\bar{M}_{w} / \bar{M}_{n}$} & \multirow{2}{*}[\alpha]{$_{\mathrm{D}}^{25}$} \\
\hline \multirow{2}{*}{$\begin{array}{l}\text { Feed } \\
100\end{array}$} & \multirow{2}{*}{$\begin{array}{c}\text { Calcd }^{\mathrm{a}} \\
-^{\mathrm{b}}\end{array}$} & & & & & & & \\
\hline & & & 59.5 & $\mathrm{~S}$ & 279.1 & 1.02 & 2.26 & $-^{\mathrm{c}}$ \\
\hline & & & 100.9 & \multirow[b]{2}{*}{$\mathrm{S}$} & 260.8 & \multirow[b]{2}{*}{2.52} & \multirow[b]{2}{*}{1.90} & \multirow[b]{2}{*}{+5.40} \\
\hline 90 & 92.8 & $\mathrm{~g}$ & & & & & & \\
\hline & & $\alpha$ & 107.9 & $\mathrm{~S}$ & 258.9 & \multirow{2}{*}{2.64} & \multirow{2}{*}{2.41} & \multirow{2}{*}{+10.6} \\
\hline & & & 102.7 & \multirow[b]{2}{*}{$\mathrm{N}$} & 211.6 & & & \\
\hline 70 & 70.6 & $\mathrm{~g}$ & & & & 3.37 & 2.51 & +17.8 \\
\hline & & & 103.0 & \multirow[b]{2}{*}{$\mathrm{N}$} & 195.4 & \multirow[b]{2}{*}{1.92} & \multirow[b]{2}{*}{2.19} & \multirow[b]{2}{*}{+22.8} \\
\hline 60 & 61.1 & $\mathrm{~g}$ & & & 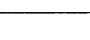 & & & \\
\hline & & & 110.5 & \multirow[b]{2}{*}{$\mathrm{N}$} & 155.2 & \multirow[b]{2}{*}{1.71} & \multirow{2}{*}{1.95} & \\
\hline 50 & 53.3 & $\mathrm{~g}$ & & & & & & +30.1 \\
\hline & 401 & & & \multirow{2}{*}{\multicolumn{2}{|c|}{118.6}} & & & \\
\hline 40 & 40.1 & g & & & & 1.77 & 1.91 & +41.2 \\
\hline 30 & 297 & & & \multicolumn{2}{|l|}{122.9} & & & \\
\hline 0 & 27.1 & g & & & & 1.50 & 1.14 & +40.9 \\
\hline 20 & 244 & $\sigma$ & & \multicolumn{2}{|l|}{128.9} & 110 & 191 & 1627 \\
\hline & & & & 113.4 & & 1.10 & 1.91 & ren \\
\hline 10 & 8.9 & $\mathrm{~g}$ & & 1110.4 & & 0.95 & 1.52 & +72.8 \\
\hline & & & & 133.8 & & & & \\
\hline 0 & 0 & $\mathrm{~g}$ & & & & 1.57 & 1.94 & +92.7 \\
\hline
\end{tabular}

${ }^{a}$ Calculated by NMR. ${ }^{b}$ Insoluble in $\mathrm{CDCl}_{3} .{ }^{\mathrm{c}}$ Insoluble in THF. g, glassy; S, smectic; N, nematic; I, isotropic. 
Table II. Phase transition temperatures, molecular weight and specific rotation of poly(CBMAB-6-co-l-MtMA)* $-x$

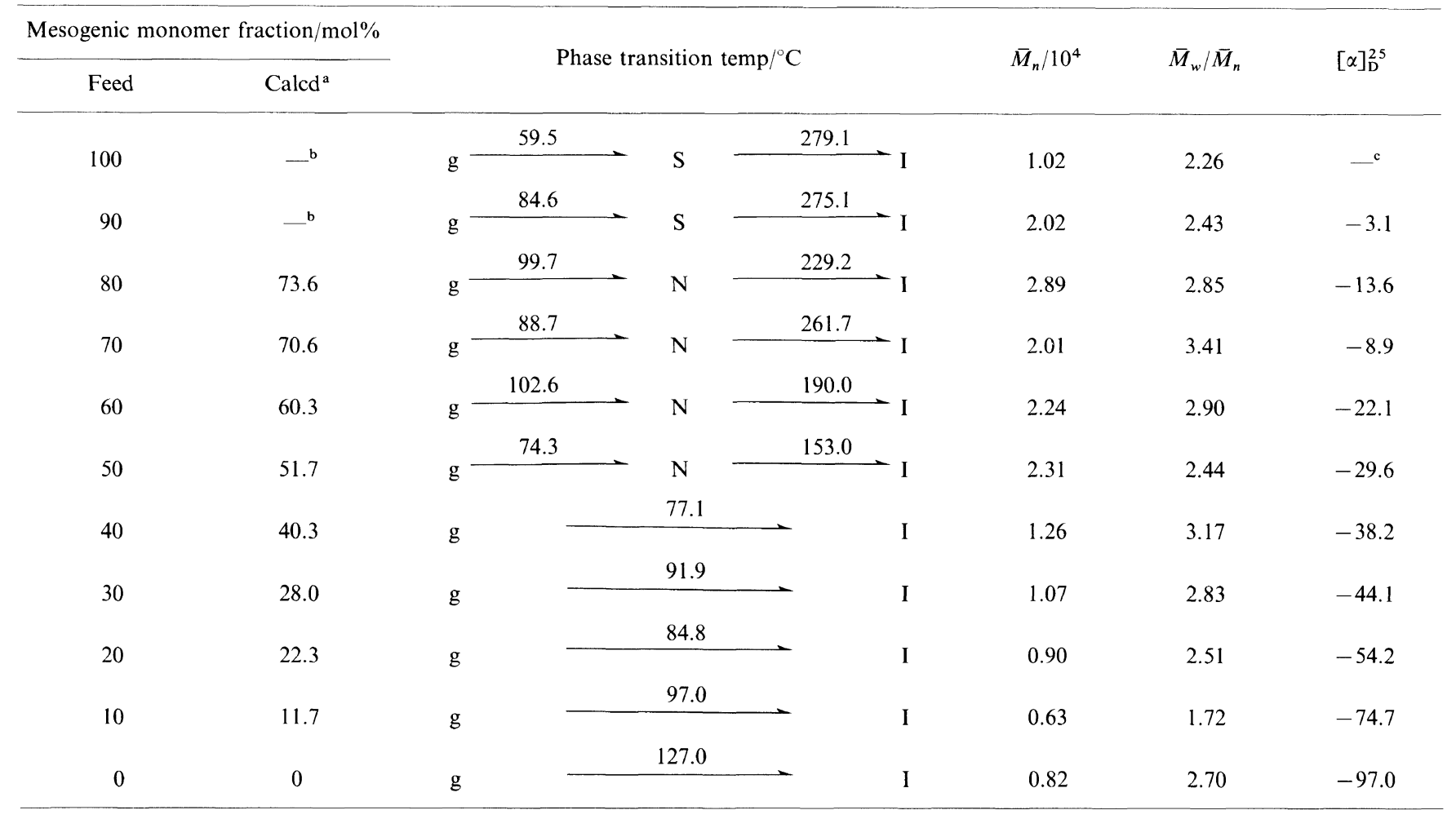

${ }^{\text {a }}$ Calculated by NMR. ${ }^{\mathrm{b}}$ Insoluble in $\mathrm{CDCl}_{3} .{ }^{\mathrm{c}}$ Insoluble in THF. g, glassy; S, smectic; N, nematic; I, isotropic.

Table III. Phase transition temperatures, molecular weight and specific rotation of poly(CBMAB-6-co-2-MBMA)* $-x$

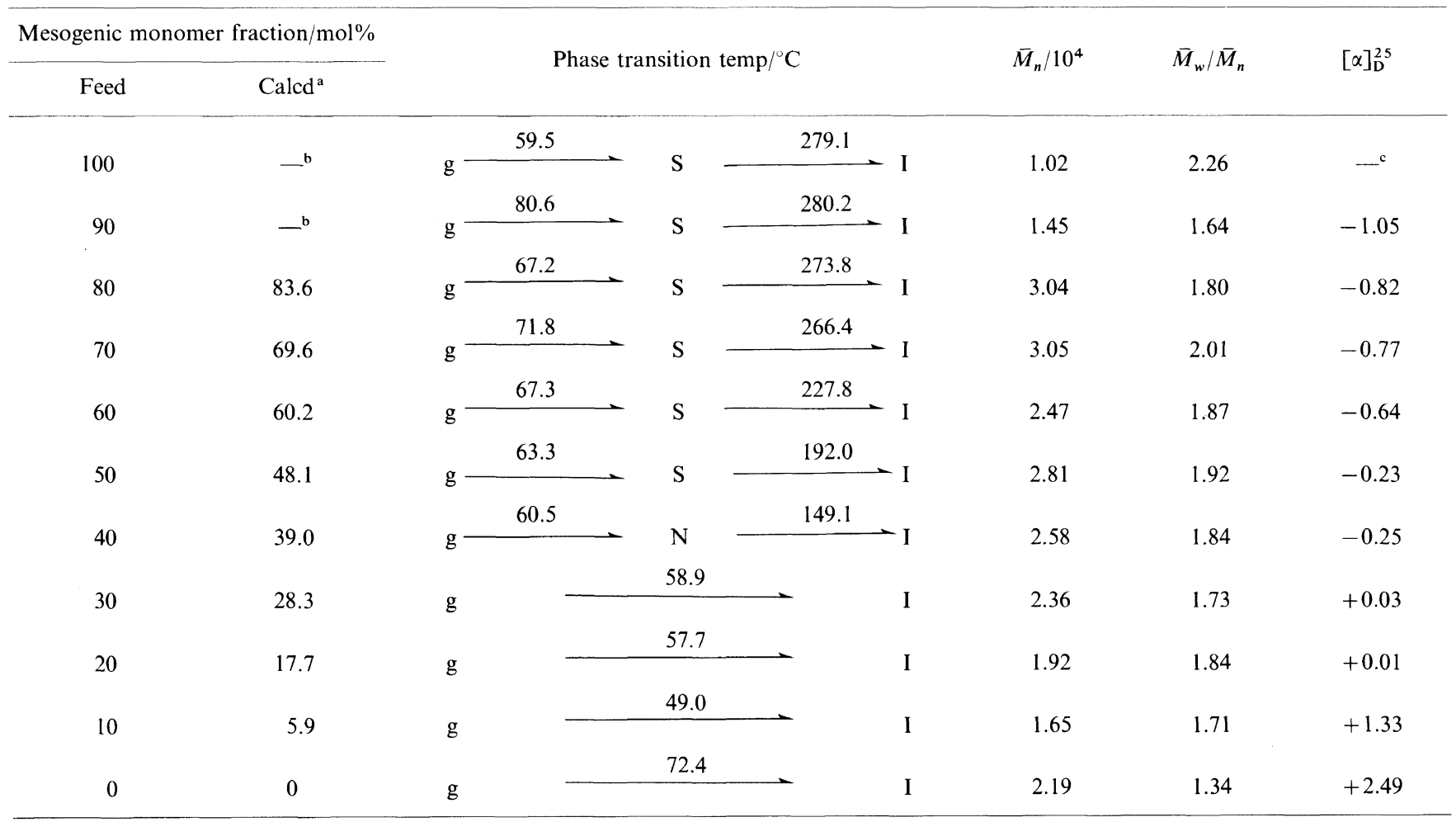

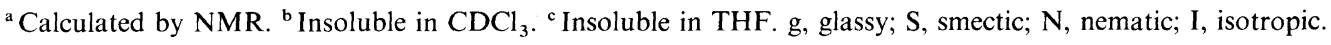

the menthyl group. The steric hindrance of the 2-methyl butyl group would be smaller than that of the menthyl group. The 2-methyl butyl group would not interfere with the orientation of the mesogenic group compared with the menthyl group.

Polym. J., Vol. 29, No. 4, 1997
However, in the X-ray diffraction patterns for poly(CBMAB-6-co-2-MBMA)* - 39.0 and poly(4-cyanobiphenyl-4'-[4-(6-methacryloyl hexyloxy)benzoate]-co- $(d$ and $l 1-$ methyl methacrylate)* (poly(CBMAB-6-co- $d$ and $l$-MtMA)*) with less than a 70 or $80 \mathrm{~mol} \%$ mesogenic 


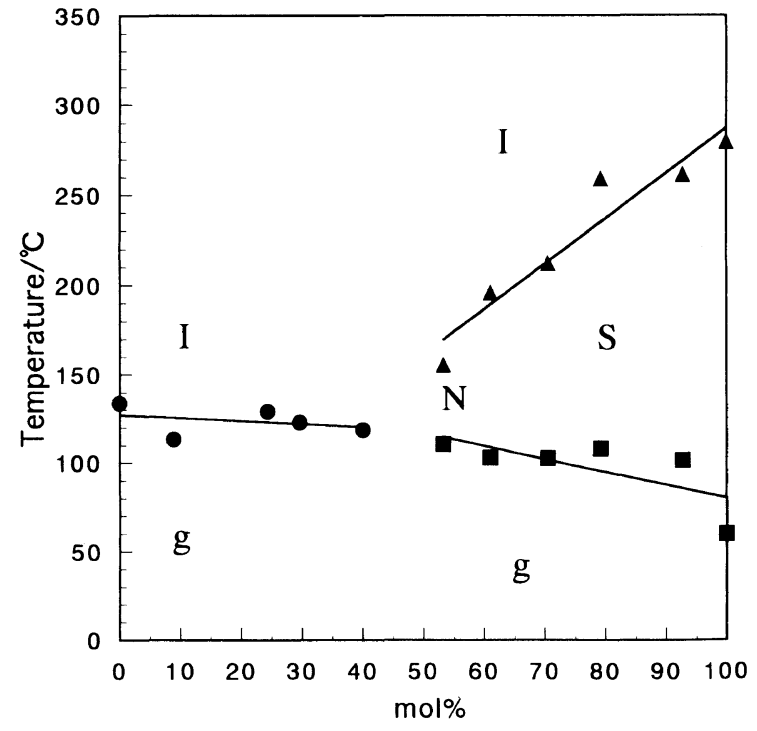

Figure 1. Phase transition temperatures as a function of mesogenic group fraction in poly(CBMAB-6-co- $d$-MtMA $)^{*}-x$. g, glassy; $\mathrm{S}$, smectic; N, nematic; I, isotropic.

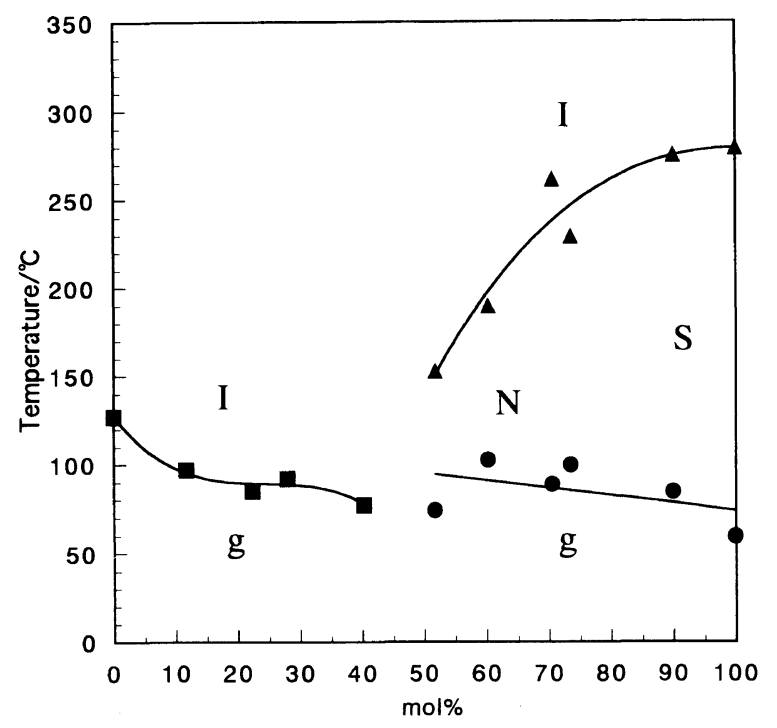

Figure 2. Phase transition temperatures as a function of mesogenic group fraction in poly(CBMAB-6-co-l-MtMA)*-x. g, glassy; $\mathrm{S}$, smectic; $\mathrm{N}$, nematic; I, isotropic.

group fraction, only one broad peak in the wide angle was observed, thus showing a nematic phase for these copolymers.

The copolymers with a 2-methyl butyl group exhibited schlieren and focal conic textures. The focal conic texture was observed only for poly(CBMAB-6-co-2-MBMA)*90 as shown in Figure 5. In contrast, no typical textures for the copolymers containing the menthyl group as a chiral moiety were observed. The difference in the textures for the copolymers occurs from the difference in the chemical structure of the chiral moieties.

We investigated the mesomorphic properties for the copolymers prepared by copolymerization of a mesogenic monomer containing no flexible spacer with a chiral monomer as shown in Figure 6(A) ${ }^{5}$ The copolymers with no flexible spacer exhibited the induced cholesteric phase regardless of the chemical structure of the chiral moiety. In contrast, the copolymers with a flexible spacer

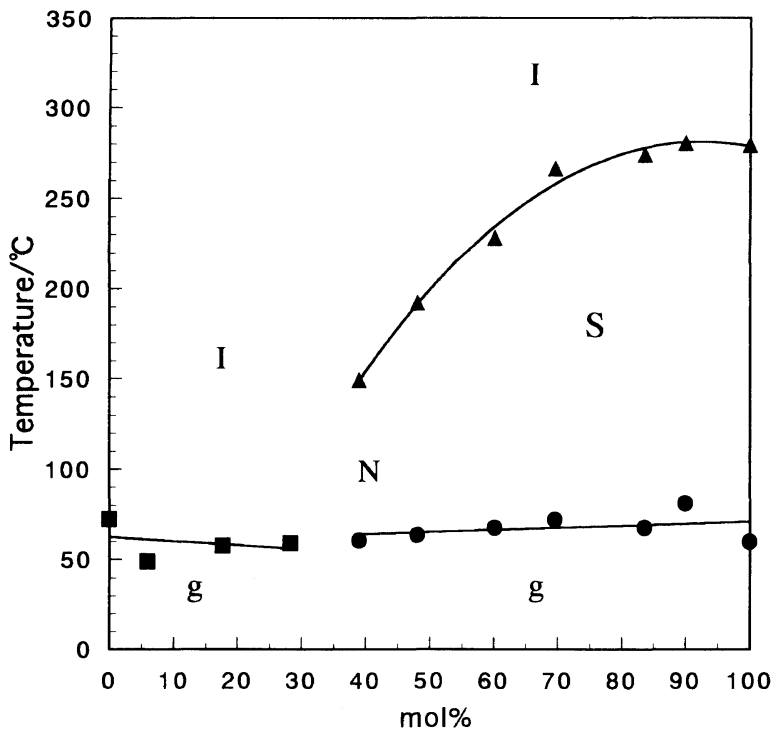

Figure 3. Phase transition temperatures as a function of mesogenic group fraction in poly(CBMAB-6-co-2-MBMA)*-x. g, glassy; $\mathrm{S}$, smectic; $\mathrm{N}$, nematic; I, isotropic.

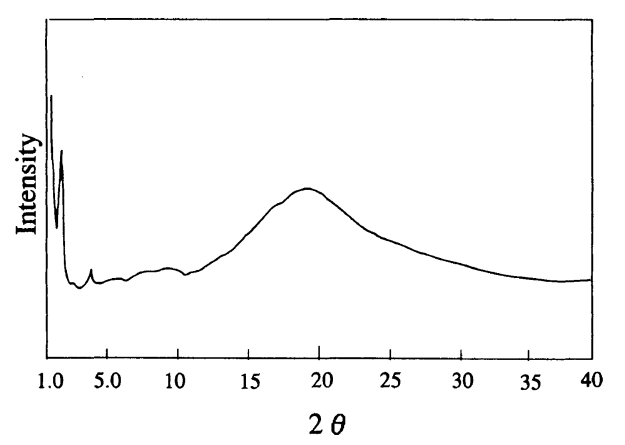

Figure 4. X-Ray diffraction pattern of poly(CBMAB-6-co-2-MBMA)* -69.6 .

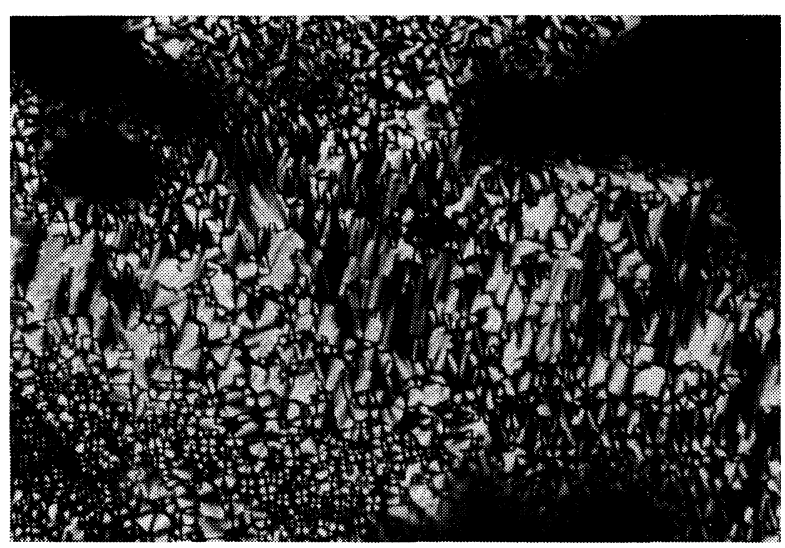

Figure 5. Optical texture of poly(CBMAB-6-co-2-MBMA)*-90 at $178.1^{\circ} \mathrm{C}$.

did not exhibit the induced cholesteric phase. The introduction of a flexible spacer separated the mesogenic group and chiral moiety with regard to the position in the copolymer as shown in Figure 6(B). The separation of the mesogenic group and chiral moiety resulted in no exhibition of the induced cholesteric phase. To examine the influence of the positioning of the mesogenic group and the chiral moiety on the mesomorphic properties of the copolymers, the mesomorphic properties for the 
(A)

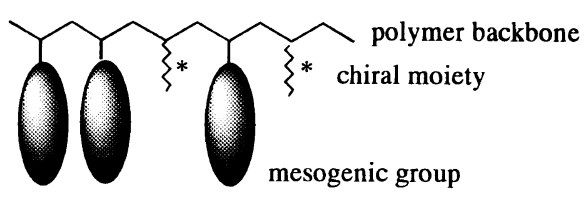

(B)
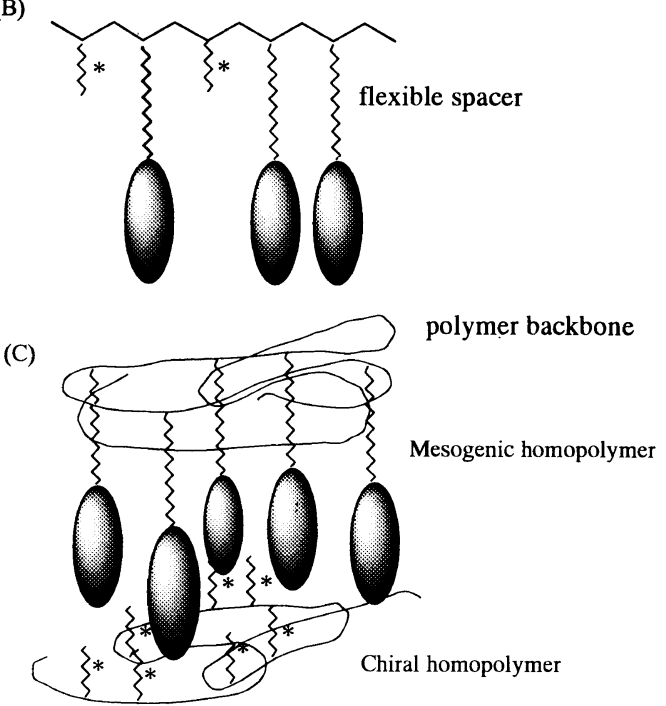

Figure 6. Schematic model of the copolymer with no flexible spacer (A), copolymer with a flexible spacer (B), and homopolymer mixture (C).

mixture of the mesogenic homopolymer with the chiral homopolymer were investigated. Close positioning of the mesogenic group and the chiral moiety would occur in the mixture as shown in Figure 6(C). A mixture of the mesogenic homopolymer and chiral homopolymer did not exhibit the induced cholesteric phase. Therefore, the induced cholesteric phase appeared only in the polymer containing the close linkage of the mesogenic group and the chiral moiety through the covalent bond. In other words, the induced cholesteric phase was only obtained by the copolymerization of the mesogenic monomer with no flexible spacer and the chiral monomer.

We examined the mesomorphic temperature range for the copolymers. The mesomorphic temperature range for all copolymers significantly decreased with decreasing fraction of the mesogenic monomer. The mesomorphic temperature range of the copolymer with $d$-menthyl moieties was nearly equal to that of the copolymer with $l$-menthyl moieties. The poly(CBMAB-6-co-l-MBMA)* $-x$ is superior to the poly(CBMAB-6-co-l-MtMA)* $-x$ with regard to the mesomorphic temperature range. The difference in the mesomorphic temperature range occurred due to the difference in the structure (steric size) of the chiral moiety. The menthyl moieties would interfere with the orientation of the mesogenic groups in the copolymers.

The phase transition temperatures of the copolymers containing a flexible spacer were lower compared with those of the copolymers with no flexible spacer. ${ }^{5}$ Thus, a flexible spacer introduced between the polymer backbone and the mesogenic group lowered the phase transition temperatures of the copolymers and stabilized the mesomorphic temperature range of the copolymers; however, no exhibition of the induced cholesteric phase for the copolymers was observed.

\section{CONCLUSION}

The copolymers prepared by copolymerization of mesogenic monomers greater than $40-50 \mathrm{~mol} \%$ with the chiral monomer exhibited smectic and nematic phases regardless of the chemical structure of the chiral moiety. The induced cholesteric phase was not observed for the copolymers with a flexible spacer.

The mesomorphic temperature range for the copolymers with the 2-methyl butyl group was wider than that of the copolymers with the $d$-and $l$-menthyl group. The mesomorphic temperature range of the copolymers with a flexible spacer was wider than that of the copolymers containing no flexible spacer. Thus, introduction of a flexible spacer into the copolymers enhanced the mesomorphic temperature range of the copolymers compared with that of the copolymers with no flexible spacer. The copolymers with a flexible spacer did not exhibit the induced cholesteric phase.

\section{EXPERIMENTAL}

\section{Materials}

The mesogenic monomer was synthesized according to Scheme 1. The synthesis of chiral monomers is described elsewhere. ${ }^{5}$

4-(6-Hydroxyhexyloxy)benzoic Acid (1). KOH (45.0 g, $0.686 \mathrm{~mol}$ ) aqueous solution was added dropwise to ethanol solution of 4-hydroxybenzoic acid $(23.7 \mathrm{~g}, 0.171$ $\mathrm{mol}$ ). Then a small amount of potassium iodide, tetra $n$-butyl ammonium bromide $(5.5 \mathrm{~g}, 0.0171 \mathrm{~mol})$ and 6 chloro-1-hexanol $(25.7 \mathrm{~g}, 0.188 \mathrm{~mol})$ were added to the mixture. The reaction mixture was refluxed for 86 hours. After concentration of the reaction mixture, the residue was poured into a solution of hydrochloric acid. The obtained precipitate was washed with water and purified by recrystallization from ethanol. The product was obtained in a $31.0 \%$ yield.

IR (Nujol) v $3332(\mathrm{OH}), 1670(\mathrm{C}=\mathrm{O}), 2634,2598$, $2530(\mathrm{COOH}), 1602,1511$ (Ar).

4-(6-Methacryloyloxyhexyloxy)benzoic Acid (2). A mixture of 4-(6-hydroxyhexyloxy)benzoic acid (3) $(25.4 \mathrm{~g}, 0.107 \mathrm{~mol})$, methacrylic acid $(100 \mathrm{ml})$, chloroform $(350 \mathrm{ml})$, a small amount of hydroquinone and a small amount of $p$-toluensulfonic acid was heated at $73^{\circ} \mathrm{C}$ for 21.5 hours to remove produced water. The reaction mixture was then concentrated, and the residue was washed with water. The solid obtained was purified by recrystallization from ethanol. The product was obtained in a $97.5 \%$ yield.

IR (Nujol) v 2671, $2563(\mathrm{COOH})$, 1716, 1683 (COO), $1632(\mathrm{C}=\mathrm{C}), 1606,1512(\mathrm{Ar})$.

4-Cyanobiphenyl-4'-[4-(6-methacryloyloxyhexyloxy)benzoate] (3: $C B M A B-6$ ). In an atmosphere of nitrogen, an anhydrous tetrahydrofuran solution of $N^{\prime}, N^{\prime}$-dicyclohexylcarbodiimide $(7.6 \mathrm{~g}, 0.037 \mathrm{~mol})$ was added to an anhydrous solution of 4-(6-methacryloyloxyhexyloxy)benzoic acid (4) $(9.4 \mathrm{~g}, 0.0308 \mathrm{~mol}), 4$-cyano-4'-hydroxybiphenyl $(5.0 \mathrm{~g}, 0.0257 \mathrm{~mol})$ and 4-(dimethylamino)pyridine $(0.5 \mathrm{~g}, 0.0037 \mathrm{~mol})$. The reaction mixture was stirred for 20 hours. The precipitate was removed by filtration 
and the filtrate was concentrated. Column chromatography (eluent chloroform: hexane $=1: 1$ ) was used to obtain the crude product with an $R f$ value of more than 0.5 on thin-layer chromatogram. The crude product was purified by washing with methanol. The product was obtained in a $73.4 \%$ yield.

NMR $\left(\mathrm{CDCl}_{3}\right) \delta 1.3-2.0(\mathrm{~m}, 8 \mathrm{H}), 2.0(\mathrm{~s}, 3 \mathrm{H}), 4.2(\mathrm{~m}$, $4 \mathrm{H}), 5.6,6.2(\mathrm{~s}, 1 \mathrm{H}), 7.0-8.4(\mathrm{~m}, 12 \mathrm{H})$.

IR (Nujol) v $2229(\mathrm{CN}), 1722,1708$ (COO), 1635 $(\mathrm{C}=\mathrm{C}), 1603,1510(\mathrm{Ar})$.

Polymerization. The copolymers with a flexible spacer were prepared by copolymerization of the mesogenic monomer and the chiral monomer in sealed ampoules with $2.5 \mathrm{~mol} \%$ (2-methylbutyl chiral monomer) or 5.0 mol\% (menthyl chiral monomer) $\alpha, \alpha^{\prime}$-azobisisobutyronitrile in anhydrous $N, N^{\prime}$-dimethylformamide at $60^{\circ} \mathrm{C}$ for 10 hours. The copolymer was obtained by reprecipitation with methanol. The copolymer was dissolved in tetrahydrofuran and reprecipitated with methanol twice. The copolymer was dried at $40^{\circ} \mathrm{C}$ under vacuum.

\section{Characterization}

${ }^{1} \mathrm{H}$ NMR was performed with a JEOL JPN-PMX60 spectrometer using $\mathrm{CDCl}_{3}$ as the solvent. Infrared spectra were recorded on a JEOL JIR-100 spectrometer. Spectra were collected at $4 \mathrm{~cm}^{-1}$ resolution. DSC measurements were conducted with a Mettler 3000 series. Optical microscopy was performed on a Nikon polarizing optical microscopy equipped with a Mettler FP80 controller and a FP82 hot stage. Gel permeation chromatography (GPC) was carried out with a Tosoh HLC-8020 instrument using chloroform as the eluent, equipped with four columns (TSK gel $\mathrm{G} 4000 \mathrm{H}_{\mathrm{HR}}, \mathrm{G} 3000 \mathrm{H}_{\mathrm{HR}}, \mathrm{G} 2000 \mathrm{H}_{\mathrm{HR}}$, and $\mathrm{G} 2000 \mathrm{H}_{\mathrm{HR}}$ ). The instrument was calibrated with a polystyrene standard. Specific rotation was carried out with JASCO DIP-370 digital polarimeter using tetrahydrofuran as the solvent.

\section{REFERENCES}

1. A. Blumstein and S. Vilasagar, Mol. Cryst. Liq. Cryst. Lett., $\mathbf{7 2 ,}$ $1(1981)$.

2. D. V. Luyen, L. Liebert, and L. Strzelecki, Eur. Polym. J., 16, 307 (1980).

3. E. Chiellini and G. Galli, Makromol. Chem., Rapid Commun., 4, 285 (1983).

4. H. Finkelmann, J. Koldehoff, and H. Ringsdorf, Angew. Chem. Int. Ed. Engl., 17(12), 935 (1978)

5. K. Nomura, T. Mihara, K. Funaki, and N. Koide, submitted for publication. 